\title{
Philip Manow
}

\section{Max Weber und die Demokratie}

\section{Einleitung}

Für den »späten « Max Weber werden Fragen legitimer politischer Herrschaft vordringlich: Kann sich die bürgerliche Gesellschaft selbst regieren? ${ }^{1}$ Er selbst unternimmt am Beginn der Weimarer Republik einen schnell scheiternden Versuch der aktiven politischen Karriere (für die DDP) und bezieht in einer Aufsehen erregenden Aufsatzserie in der Frankfurter Zeitung im November 1918 zur politischen Neuordnung Deutschlands Stellung. Schließlich übt er auch als Mitglied des Verfassungsausschusses unter Hugo Preuß einen wichtigen Einfluss auf die Weimarer Verfassung aus. ${ }^{2}$ Insbesondere aber mit Politik als Beruf ${ }^{3}$ und mit Parlament und Regierung im neugeordneten Deutschland ${ }^{4}$ verfasst er zwei weit beachtete, auch weit über den unmittelbaren Anlass hinaus wirkende Diagnosen. Zumindest was die erste Schrift angeht, hat Weber in den Umbruchjahren 1918 und 1919, kurz vor seinem Tod, einen Klassiker der Politischen Theorie formuliert. Politik als Beruf und die dort entworfene Konzeption von Demokratie sollen im Folgenden besonders im Vordergrund stehen.

Ich werde dabei im Wesentlichen folgende These ausarbeiten: Weber trifft seine politische Diagnose in einer besonderen gesellschaftlichen, politischen Umbruchsituation - und sie gewinnt für uns, die wir uns wiederum in einer Umbruchsituation befinden, daher besondere Relevanz. Spezifischer formuliert: Weber steht am Anfang eines Entwicklungsbogens - dem Beginn einer funktionierenden repräsentativen Demokratie in Deutschland -, und wir erleben heute zumindest einen Moment ihrer grundsätzlichen Krise. ${ }^{5}$ Dies macht die Auseinandersetzung mit dem späten Weber, dem politischen Diagnostiker, für uns so wichtig.

\section{Demokratie ohne Unterleib?}

Wenn man Politik als Beruf heute erneut liest, so hat der Text natürlich nichts von seiner Faszination verloren. Der "Staat als Monopol legitimer physischer Gewaltsamkeit«, die drei Prinzipien der Herrschaftslegitimation (Tradition, Legalität,

1 Dieser Text ist die überarbeitete Version eines Vortrags, der am 21. September $2020 \mathrm{im}$ Rahmen der Ringvorlesung »Entfesselt: Kapitalismus, Religion, Demokratie« aus Anlass des 100. Todestages von Max Weber an der Universität Heidelberg gehalten wurde.

2 Hierzu detailliert Mommsen 1974 [1959].

3 Weber 1992 [1918].

4 Weber 1988 a [1917].

5 Manow 2020. 
Charisma), Verantwortungs- versus Gesinnungsethik, Weltanschauungs- versus Stellenjägerparteien, Leben für die Politik und Leben von der Politik, die Politik als das lange Bohren harter Bretter - die hohe Anzahl zentraler Definitionen und Begrifflichkeiten, wie sie in das intellektuelle Arsenal der Politikwissenschaft und teilweise sogar in den allgemeinen Sprachschatz aufgenommen worden sind, zeugt von Webers bis heute spürbar machtvollem analytischen Zugriff. Kontextualisiert fällt vielleicht zusätzlich noch die spezifische Mischung aus Nicht-Pathos einerseits und Pathos dieses Nicht-Pathetischen andererseits auf. Man muss sich ja vergegenwärtigen, dass diese Schriften verfasst werden in einem Moment des totalen Zusammenbruchs einer ganzen Gesellschaft und der völligen Delegitimierung ihrer politischen Ordnung. Vor diesem Hintergrund erscheinen diese Analysen dann doch erstaunlich gefasst, aber dieses Gefasst-Sein entspringt natürlich einer eigenen Attitüde des Heldischen, ist Teil der »Verhaltenslehren der Kälte « - vornehmlich gespeist aus Nietzsche-Lektüre -, die Helmuth Lethen für das Deutschland der Zwischenkriegszeit analysiert hat. ${ }^{6}$ Das ist die Rhetorik der »Polarnacht von eisiger Finsternis und Härte «, durch die man nun erstmal durchmüsse. Aber das ist für unseren Zusammenhang vielleicht nicht von primärem Interesse.

Abgesehen von diesem Eindruck ergibt sich ein zweiter, und der ist weniger faszinierend, sondern - zumindest aus heutiger Sicht - eher schockierend. Nachdem er seine klassisch gewordene Definition von moderner staatlicher Herrschaft formuliert - eben der "Staat als Monopol legitimer physischer Gewaltsamkeit «, fragt Weber: Und was macht diese Herrschaft zu einer legitimen? Er antwortet bekanntermaßen mit Verweis auf seine Herrschaftssoziologie - und das ist schockierend nicht wegen dessen, worüber er dann nachfolgend spricht, sondern wegen dessen, worüber er nicht spricht. Weber nennt als die drei Quellen der Legitimation von Herrschaft die Tradition, die Legalität - also die schlichte Regelgebundenheit und Berechenbarkeit - und das Charisma. Worüber spricht er nicht? Er spricht nirgends darüber, dass die Legitimation einer Herrschaft doch möglicherweise auch darin begründet liegen könnte, dass man ihr zugestimmt hat. Er spricht also nicht über - Demokratie.

Das Wort selbst kommt insgesamt nur elf Mal auf den knapp 80 Druckseiten vor (wenn wir Wörter wie Sozialdemokratie unberücksichtigt lassen), und es ist bei ihm kaum analytisch, sondern vorwiegend deskriptiv gewendet, meint eigentlich meistens Länder oder deren politische Systeme: die amerikanische Demokratie, die von ihr beeinflussten Demokratien, die antike Demokratie etc. Was die Demokratie genau charakterisiert, was ihre Mechanismen sind, wie sich die moderne von der antiken Demokratie unterscheidet, auf welche Voraussetzungen sie im modernen Nationalstaat trifft - nirgends finden sich dazu sonderlich systematische Gedanken. Natürlich wird die plebiszitäre Führerdemokratie aufgerufen, aber die bestimmt sich ja bei Weber nun gerade nicht dadurch, dass ihr ein in Wahlen begründetes Delegations- oder Mandatierungsverhältnis zugrunde liegt, sondern in der unbedingten Gehorsamsbereitschaft einer Gruppe aufgrund der besonderen charismatischen Qualitäten ihres Führers. "Seine Autorität bei den 
Massen und bei seinen Anhängern gründet sich nicht auf seine von diesen gebilligten sachlichen Ziele, sondern auf seine persönlichen Qualitäten; er ist weniger ihr Vertrauensmann als ihr Herr, dem sie sich im Wahlakt für die Dauer der Wahlperiode bedingungslos verschrieben haben. ${ }^{7}$ Es war Webers Überzeugung, dass »der große Politiker sich seine politische Gefolgschaft kraft seiner persönlichen charismatischen Qualifikationen zu schaffen habe, mit anderen Worten, daß Willensbildung prinzipiell von oben nach unten verlaufe und nicht von der Basis hinauf zu den gewählten Führern, sei es kraft des Prinzips der Delegierung oder der Übertragung des politischen Mandats «. ${ }^{8}$

In diesen Zusammenhang gehört die Beobachtung, dass Weber vollständig davon absieht, irgendeinen Vertreter der Politischen Theorie oder Politischen Philosophie auch nur kurz aufzurufen, bei denen Fragen der Repräsentation und der Volkssouveränität sich durchweg zentral verhandelt finden beziehungsweise politische Herrschaft üblicherweise vertragstheoretisch begründet wird, also als Resultat freier Übereinkunft: Hobbes, Pufendorf, Rousseau, Sièyes, Hegel, ... nichts. Ein einziges Mal bietet der Name Machiavelli für Weber den Anlass, zu konstatieren, dass dessen Il Principe verglichen mit dem indischen Kautaliya Arthasastra aus lang vorchristlicher Zeit ja ein völlig harmloses Machwerk sei.

Man kann fragen, wie diese offensichtliche thematische Vernachlässigung zu erklären ist: Die preußische Wahlreform wird über die gesamte Zeit des Kaiserreichs aufs Leidenschaftlichste diskutiert, sie ist eines der brennendsten politischen Themen der Zeit; 1918 wird das allgemeine Männerwahlrecht auf die Frauen ausgeweitet; mit dem Entschluss des Rats der Volksbeauftragten im Kontext der Novemberrevolution wechselt das Reichstagswahlrecht vom absoluten Mehrheitswahlrecht zur Verhältniswahl, womit sich die Funktionsbedingungen politischer Parteien von Grund auf ändern (ich komme darauf zurück); Weber selbst hatte sich zum Thema ausführlich zu Wort gemeldet ${ }^{9}$ - warum, so muss man also fragen, findet das alles überhaupt keinen Niederschlag in der in Politik als Beruf entworfenen Konzeption von Demokratie? Wenn es ihm doch zentral um Führungs-

\section{Mommsen 1974, S. 49.}

8 Mommsen 1989, S. 527. Deutlich, auch in diesbezüglichen Äußerungen in Wirtschaft und Gesellschaft, ist, dass hier die Legitimation »von oben ", von der Führergestalt her gedacht wird und nicht von unten, als Delegations- und Legitimierungsakt in Wahlen (Mommsen konstatiert Webers Entscheidung, »seine Theorie demokratischer Herrschaft nicht von den Grundpostulaten der Selbstbestimmung des einzelnen und der Volkssouveränität her zu entwickeln «; ebd., S. 526). Demokratie als Willen des Volkes fungiert dann eigentlich nur als Camouflage: »Die splebiszitäre Demokratie - der wichtigste Typus der Führer-Demokratie - ist ihrem genuinen Sinn nach eine Art der charismatischen Herrschaft, die sich unter der Form einer vom Willen der Beherrschten abgeleiteten und nur durch ihn fortbestehenden Legitimität verbirgt « (zitiert nach ebd., S. 538; Hervorhebung P.M.). Das ist nicht weit entfernt von Carl Schmitts zeitgleich entworfener Vision einer souveränen Diktatur, bei der der Diktator »aber infolge der Eigenart der nicht konstituierten, aber konstituierenden Gewalt des Volkes [...] auch seinem Auftraggeber diktiert, ohne aufzuhören, sich an ihm zu legitimieren " (Schmitt 2015 [1921], S. XIX).

9 Weber 1988 b [1917]. 
auslese geht, warum betrachtet er dann nicht den demokratischen Mechanismus der Führungsauslese - die Wahl?

Meine Vermutung lautet, dass Weber Demokratie implizit vor dem Hintergrund dessen analysierte, was er von Droysen über die attische Demokratie und von Mommsen über die Römische Republik gelernt hatte. In diesem großbürgerlichen Bildungsmilieu, vor dem Hintergrund des "antiken-orientierten « Bildungskanons des deutschen humanistischen Gymnasiums und der deutschen Universität des späten 19. Jahrhunderts, der besonders hohen Rangstellung der »klassischen Altertumswissenschaften « in der Hierarchie der Wissenschaften der Zeit, war, so vermute ich, dieses Wissen um die antike Demokratie für Weber auf eine selbstverständliche Art und Weise die Deutungsfolie (nicht die Legitimationsressource $)^{10}$ für die Analyse der Gegenwart. Aber in Athen und Rom war das Volk immer schon versammelt - und politische Mehrheiten bildeten sich in der Ekklesia oder römischen Volksversammlung mehr oder weniger spontan, kaum vororganisiert, allein durch die Überzeugungskraft der Rede einer charismatischen Persönlichkeit.

Natürlich ist das in der modernen Demokratie anders, hier agieren Parteien als zentrale Mobilisierungsakteure (die dann auch die parlamentarische Mehrheitsbildung determinieren oder zu determinieren versuchen) - und selbstverständlich widmet sich Weber diesen neuen Akteuren mit großer Aufmerksamkeit und enormem analytischen Verstand und vor dem Hintergrund der zeitgenössischen Literatur: Gaetano Mosca, Vilfredo Pareto, Moissei Ostrogorski, James Bryce, nicht zuletzt auch in Rückgriff auf die Studie von Robert Michels, seines Doktoranden, zur deutschen Sozialdemokratie. ${ }^{11}$ Aber dabei geraten allenfalls die sozialen Implikationen der Ausweitung des Wahlrechts in den Blick oder die damit einhergehende Veränderung in der Zusammensetzung des Parlaments. Wie aber die Wahl und das Wahlrecht selbst die Organisationsform Partei zentral definieren und darüber deren Handlungsrepertoire im parlamentarischen Bereich sowie die Prozesse politischer Führungsauslese bestimmen, bleibt bei Weber unberücksichtigt - mit ganz spezifischen Folgen für seine Zeitdiagnose und deren bedeutsame Nachwirkungen.

\section{Patronage, Stellenjägerei, Pfründen}

Stattdessen nähert sich Weber dem Thema quasi organisationssoziologisch, beeinflusst von seinen Studien zur Bürokratie, zum Betrieb, zum eisernen Gehäuse der Hörigkeit - aber von oben, aus staatlicher Perspektive, nicht von unten, der Mobilisierungsperspektive oder der Perspektive der spontanen Selbstorganisation kollektiven politischen Handelns. Natürlich kann es sehr hilfreich und heuristisch wertvoll sein, sich dem Thema aus dieser, wenn man so will, inkongruenten Sicht zu nähern. Und es ist dann auch auf einzigartige Weise anregend zu lesen, was

10 Vgl. Momigliano 1991 [1955]; Nippel 2008.

11 Michels 1957 [1911]. 
Weber im längeren Mittelteil des Textes ${ }^{12}$ vor allem über die Parteien, teilweise auch über die Parlamente und deren Organisationserfordernisse und Organisationslogiken zu sagen hat. Zugleich ist aber eben festzuhalten, dass dies mit spezifischen analytischen Verkürzungen und wohl auch schwerwiegenden Verzerrungen einhergeht. Man könnte es erneut an der verwendeten Begrifflichkeit verdeutlichen. Weber spricht in Politik als Beruf elf Mal von Demokratie, aber sechs Mal von Stellenjägern, zehn Mal von Patronage, elf Mal von Pfründen und Pfründern (unter anderem als schöne Wortkreation: der Staat und seine Ämter als »Pfründnerversorgungsanstalt«). 25 Mal geht es um Ämter und ihren Besitz, um Sporteln (oder Sportelpfründen), Sinekuren, Beute (sieben Mal), um die Beutepolitik der Parteien etc.

Weber nennt eine atemberaubende Zahl: Mit einem Wechsel im Präsidentschaftsamt würden in den USA auch zugleich mehrere hunderttausend öffentliche Ämter ihren Besitzer wechseln (»in den Händen des Präsidenten liegen immerhin 300.000 bis 400.000 Beamtenernennungen «13). Mich würde interessieren, ob es in der extrem reichhaltigen Weber-Sekundärliteratur irgendeinen Beleg für diese fantastische Zahlenangabe gibt - in der historisch-kritischen Gesamtausgabe seiner Werke bleibt diese Passage unkommentiert und die Zahl unbelegt. ${ }^{14}$ Ich muss gestehen, dass ich sie für unseriös halte. ${ }^{15}$ Kein US-amerikanischer Präsident, vor allem angesichts des relativ rudimentären Organisationsniveaus politischer Parteien in den Vereinigten Staaten, wäre in der Lage, auch nicht innerhalb einer gesamten präsidialen Amtszeit, 300.000 bis 400.000 Beamtenstellen mit Parteigefolgschaft zu besetzen. ${ }^{16}$ Webers wiederholte Verweise auf die Parteimaschine als

12 Weber 1992 [1918], S. 39-59.

13 Ebd., S. 50-51.

14 Vgl. Mommsen et al. 1992, S. 214.

15 Der Pendleton Civil Service Reform Act von 1883 hatte das Patronagesystem erheblich eingeschränkt, die Progressive Era die Korruption erheblich zurückgedrängt. »By 1909, almost two-thirds of the U.S. federal workforce was appointed based on merit, that is, qualifications measured by tests. " Das ist neun Jahre, bevor Max Weber Politik als Beruf schreibt. Für das Jahr 1918, in dem Weber wohl noch kaum Zugang zu aktuellen Statistiken hatte, werden 917.760 Bedienstete in der Executive Branch der Bundesregierung angegeben, eine Zahl, die offensichtlich stark durch den Krieg aufgebläht ist, denn sie sinkt in den Folgejahren dramatisch auf etwa 530.000, auf das Niveau, das auch zuvor vorherrschte: im Jahr zuvor, 1917, lag sie bei 517.000 (Bureau of Census 1949, S. 294). Ein Drittel von 517.000 ist circa 170.000 - das wäre immer noch eine sehr hohe Zahl, wenn auch deutlich unter Webers äußerst vager Schätzung von 300.000 bis 400.000 . Die Abqualifizierung des US-amerikanischen Staates, der gerade durch seine kriegswirtschaftlichen Anstrengungen und seine Truppenverlegung nach Europa Deutschlands Niederlage besiegelt hatte, als reine Dilettantenwirtschaft zeugt ebenfalls nicht gerade von einem gesteigerten Reflexionsvermögen des Verlierers im Moment der Niederlage (Schivelbusch 2001).

16 Heute, in Zeiten enorm gewachsener Staatsaufgaben, geht man von circa 4.000 Exekutivposten aus, die im Zuge eines Machtwechsels im Weißen Haus neu zu besetzen sind. Diese Prozesse ziehen sich gewöhnlich bis in die zweite Hälfte der Amtszeit eines Präsidenten. 
persönliches Belohnungs- und Patronagenetzwerk um einen »Boss « herum, konkret etwa auf die New Yorker Tammany-Hall Machine, sind in diesem Zusammenhang ganz unpassend, denn hier handelt es sich ja durchweg um lokale Phänomene, um die politisch gesteuerte Besetzung der Ämter im Bereich kommunaler »Daseinsvorsorge " (bei der Polizei, der Müllabfuhr etc.); nichts, was mit dem USPräsidentenamt und dem Parteienwettbewerb um seine Besetzung zu tun hätte, und nichts, was auf bundesstaatlicher Ebene irgendwie hätte gesteuert werden können. Diese politische Maschine ist übrigens auch ein ganz anderes Phänomen als der von Weber in seiner Funktion mit ihr gleichgesetzte electoral causus der liberalen Partei in Großbritannien im letzten Drittel des 19. Jahrhunderts. ${ }^{17}$ Weber vergleicht hier also völlig heterogene Dinge. Man fragt sich, ob sich in diesen Passagen nicht eine ganz spezifische Obsession artikuliert, die sicherlich nicht nur die private von Max Weber ist, sondern Ausdruck auch eines in Deutschland zeitgenössisch sehr ausgeprägten und dann leider noch lange nachwirkenden AntiParteienaffekts. ${ }^{18}$

Abgesehen von dubiosen Zahlenangaben führt das letztlich bei Weber dann auch zu eher verstiegenen Einschätzungen: »Alle Kämpfe sind nicht nur Kämpfe um sachliche Ziele, sondern vor allem auch: um Ämterpatronage « - das mag in dieser Zuspitzung noch gerade hingehen; dann aber: "Zurücksetzungen in der Anteilnahme an den Ämtern werden von den Parteien schwerer empfunden als Zuwiderhandlungen gegen ihre sachlichen Ziele. ${ }^{19}$ Ist das wirklich so - gerade angesichts Webers eigener Begriffsschöpfungen "Weltanschauungspartei« und »Gesinnungspolitik «?20 In diesen Einschätzungen macht sich meines Erachtens nicht nur ein Anti-Parteienaffekt bemerkbar, sondern ein Defekt der Analyse selbst. Es zeigen sich die Konsequenzen, dass Weber sich für Demokratie allenfalls zur Hälfte zu interessieren scheint. In Parallele zu seiner Kapitalismusanalyse in der Protestantischen Ethik, in der - wie Jürgen Kaube treffend angemerkt hat ${ }^{21}$ die asketischen Produzenten prominent vorkommen, ihr notwendiges Komplement, die hedonistischen Konsumenten, hingegen gar nicht, kann man für unseren Zusammenhang zum gleichen Befund gelangen: Wir sind mit einer politischen Theorie konfrontiert, in der Politiker und Parteien als Produzenten von Politik vorkommen, Wähler als deren Konsumenten hingegen nicht. An einer Stelle des Textes vergleicht Weber den eigentlichen demokratischen Souverän, der für ihn offensichtlich nur ein »von Fachbeamten regiertes Volk« darstellen kann, mit

17 Dies war historisch nur eine Organisationsform, um das Verhalten liberaler Wähler über verschiedene Wahlkreise hinweg zu koordinieren, und hatte mit politischer Patronage nichts zu tun. Mommsen merkt zurückhaltend an, dass das »Beutesystem [...] in seiner Bedeutung für das Funktionieren der amerikanischen Präsidialverfassung stark überschätzt wurde« (Mommsen 1974 [1959], S. 372).

18 Gusy 1993.

19 Weber 1992 [1918], S. 20.

20 Ebenso überspitzt: Im Hinblick auf ihre Interessen an der Versorgung mit Ämtern würden Parteien für ihre »Gefolgschaft immer mehr Mittel zum Zweck« (ebd., S. 21).

21 Vgl. Kaube 2014, S. 186. 
einer ohnmächtigen Aktionärsversammlung, die gegenüber jeder Betriebsführung ebenfalls völlig einflusslos bleiben müsse. ${ }^{22}$ Weber bringt es fertig, beide Pole seiner Analogie zugleich grob verzerrt darzustellen.

Das wird natürlich im Kontext seiner grundsätzlicheren Interpretation des okzidentalen Rationalismus verständlich, seiner Betonung der Dominanz des rationalen Betriebs und der disziplinierten Lebensführung, der Bedeutung von Regelhaftigkeit, Berechnung, Disziplin, von Bürokratie als Schicksal, des modernen Anstaltsstaats, des eisernen Gehäuses der Hörigkeit etc. Der Text setzt ja ein mit der These, dass die Trennung der Produzenten von den Produktionsmitteln, die den modernen Kapitalismus kennzeichnet, nicht weniger für den modernen bürokratischen Staat charakteristisch sei. Beide Male dominiert also, nach Weber, der moderne Management-Typus, der im Bereich des Staates der moderne Fachbeamte ist. Die Parteien tauchen in dieser Sicht dann auf als Akteure, die diesen Rationalisierungsvorgang zumindest teilweise wieder rückgängig $\mathrm{zu}$ machen suchen - indem ihre Beutepolitik sich diese Produktionsmittel von Herrschaft anzueignen versucht. Für Weber bricht damit aber durch die Politik sozusagen auch das Irrationale wieder in einen "eigentlich « rational gewordenen Staatsbetrieb ein.

Insofern haben wir es bei ihm mit einer seltsamen Ungleichzeitigkeit der Analyse zu tun. Das politische System wird mit den Schemata der deutschen Altertumswissenschaft von Droysen und (Theodor) Mommsen analysiert: Hier liegt die Hauptaufmerksamkeit auf den Tribunen- oder Demagogenqualitäten frei agierender politischer Führer. ${ }^{23}$ Der Staat hingegen wird mit der Begrifflichkeit von Karl Marx analysiert, und für Weber wird die marxistische Vorstellung einer Expropriation der Expropriateure prägend: Nachdem vermittels des modernen Staatsbetriebs der Feudalherrscher entmachtet worden ist, ihm dank des meritokratischen Prinzips die Herrschaft über die Ämter genommen wurde, tauchen mit den Parteien neue politische Akteure auf, die nun die Herrschaft über den modernen, bürokratischen, von Fachbeamten geleiteten Staat anstreben. Man wird sich das wohl nicht - wie bei Wolfgang Mommsen ${ }^{24}$ - als eher harmonisches oder komplementäres Verhältnis vorstellen dürfen, bei der eine auf die Parteimaschine gestützte politische Führerpersönlichkeit eine Staatsverwaltung dynamisiert, während das Fachbeamtentum dessen politisches Charisma rationalisiert und diszipliniert. Denn die parteipolitische Ämterpatronage, auf der das Funktionieren des einen, politischen Pols basieren soll, würde die Funktionslogik des anderen, bürokratischen Pols systematisch zwangsläufig unterminieren.

22 Weber 1992 [1918], S. 26-27.

23 Referenzpunkt wird hier unmittelbar Perikles als Oberstratege, wie er »die souveräne Ekklesia des Demos von Athen « leitete (Mommsen et al. 1992, S. 191). Schon in der Entfaltung der charismatischen Herrschaft kommt bei Weber »die Hingabe an das Charisma des Propheten oder des Führers im Kriege oder des ganz großen Demagogen in der Ekklesia oder im Parlament « zur Sprache (ebd., S. 161; Hervorhebung P.M.).

24 Mommsen 1989. 


\section{Die plebiszitäre Führerdemokratie als Erblast}

Hätte Weber auch die Wähler in den Blick genommen, dann hätte das wohl eine Unterscheidung wie die von "Stellenjägerparteien « einerseits und »Weltanschauungsparteien " andererseits stark relativiert. Im Jargon der heutigen Politikwissenschaft finden solche Unterscheidungen ihr fernes Echo, wenn danach differenziert wird, ob Parteien eher policy- oder eher office-seeking seien. ${ }^{25}$ Aber egal, ob sie das eine oder das andere sind, müssen sie doch durchweg vote-seeking sein, um entweder ihre Amts- oder ihre Politikinteressen durchsetzen zu können. Eben das sichert ja in der funktionierenden Demokratie, dass die möglicherweise rein partikularen Motivationen der politisch Handelnden an die substantiellen Interessen der ihnen zur Macht Verhelfenden, also der Wählerschaft, systematisch rückgebunden werden. Im Schumpeterianischen Demokratieverständnis - als Wettbewerb zwischen politischen Eliten um die Besetzung der führenden Staatsämter mit den Wählern, die hier den Zuschlag geben - ist dieser Zusammenhang viel klarer und viel kühler gesehen und gefasst. ${ }^{26}$

Als Zwischenfazit lässt sich also der problematische Befund festhalten, dass Weber in der Verbindung von seiner Herrschaftssoziologie und ihrer - laut Eigenanspruch - alle Legitimationsformen erschöpfenden Trias aus Tradition, Legalität und Charisma mit der politischen Theorie, wie er sie in seinen späten Schriften entwirft, nur eine einzige Herrschaftslegitimation kennt, die nicht auf die in dem »faktischen Bestehen von Herrschaft begründete Hinnahme der Herrschaftsordnung als einer unabänderlichen " hinausläuft, ${ }^{27}$ und das ist die charismatische Herrschaft. Diese ist wiederum bei ihm nur äußerst lose, wenn überhaupt, demokratisch fundiert. Entworfen wird allenfalls eine Schwundstufe von parlamentarischer Demokratie, in »deren Mittelpunkt die große, letzten Endes nur sich selbst und ihren selbstgewählten >letzten Werten` verpflichtete Führergestalt " steht. ${ }^{28}$

Das ist eine eigenartig verzerrte Wahrnehmung der Funktionsweise demokratischer Delegation. Sie sollte sich auch als wenig hilfreich in dem Verständnis dessen erweisen, was sich in Deutschland nun nach 1918 zunächst politisch herausbildete, hatte jedoch selbst einen durchaus verhängnisvollen Einfluss auf eben dieses weitere Geschehen - als Stichwort sei nur genannt die Stellung des Reichspräsidenten nach Artikel 48 (mit seinen umfassenden Notstandsrechten) der Weimarer Reichsverfassung (hierzu gehören aber auch der Artikel 25: Recht zur Reichstagsauflösung; der Artikel 53: Recht zur Ernennung und Entlassung des

25 Müller, Strom 1999.

26 Schumpeter 1993 [1942]. Statt der bei Weber (1992 [1918], S. 51) erneut normativ stark eingefärbten Einschätzung: »Daß ganz gesinnungslose Parteien einander gegenüberstehen, reine Stellenjägerorganisationen, die für den einzelnen Wahlkampf ihre wechselnden Programme je nach der Chance des Stimmenfangs machen [...].«

27 Mommsen 1989, S. 538.

28 Ebd., S. 532. »Über dem Parlament steht also damit der faktisch plebiszitäre Diktator, der die Massen vermittels der >Maschine hinter sich bringt, und für den die Parlamentarier nur politische Pfründner sind, die in seiner Gefolgschaft stehen. « (Weber 1992 [1918], S. 49) 
Reichskanzlers, auf dessen Ersuchen hin auch von Reichsministern; der Artikel 47: Oberbefehl über die Streitkräfte etc.). ${ }^{29}$ Hier ist nicht der Platz, die ja bekannte Geschichte nochmals nachzuerzählen, wie es dazu kommen konnte, dass die Weimarer Verfassung in letztlich fataler Weise dieses Neben- und Gegeneinander von Parlament und Präsident festschrieb. ${ }^{30}$ Weber ist natürlich nicht der einzige, der dafür verantwortlich zu machen ist. Insbesondere Hugo Preuß, aber auch Richard Redslob und andere wären hier zu nennen. Aber in seiner regen Veröffentlichungstätigkeit und auch als Experte vor dem Verfassungsausschuss hat sich Weber mit seinem erheblichen Gewicht und seiner ganzen Wortgewalt, geleitet von seiner Vorstellung einer plebiszitären Führerdemokratie, für diese letztlich verhängnisvolle Konstellation eingesetzt.

In diesem Kontext addiert sich bei ihm Fehleinschätzung zu Fehleinschätzung, wenn er etwa in der 1918 eingeführten Verhältniswahl nur das Instrument zur Bildung eines »Banausenparlaments « sehen kann, das »unfähig [sei,] in irgendeinem Sinne eine Auslesestätte politischer Führer darzustellen ", 31 während er zugleich meint, ein direkt gewählter Reichspräsident könne der »Anlass zur Führungsauslese und damit einer Neuorganisation der Parteien « sein. ${ }^{32}$ Es ging Weber letztlich darum, ein »Gegengewicht « zu dem in seinen Augen »in schweren Mißkredit« geratenen Parlament zu bilden, ${ }^{33}$ aber das hatte etwas von einer selbsterfüllenden Prophezeiung an sich, weil in einer solchen Konzeption dem Parlament geringere Entwicklungsmöglichkeiten zugestanden wurden, es weniger politisch in die Pflicht genommen wurde.

Bei aller retrospektiven (und daher in gewisser Weise wohlfeilen) Kritik ist aber doch festzuhalten, wie analytisch kraftvoll erneut sein Zugang ist. Weber diagnostiziert den Übergang von der Aristokratenpartei zur Honoratiorenpartei, ein Typus von Partei, der sich dann selbst herausgefordert sieht durch einen neuen Parteientypus, den Weber insbesondere in den Vereinigten Staaten und in England zu erkennen meint, die "politische Maschine " um einen »Boss « oder election agent, oder als Variante die Funktionärspartei, wie sie die deutsche Sozialdemokratie verkörpert. Das ist analytisch sehr scharf, prägend für nachfolgende Analysen bis in die Gegenwart hinein, die ganz übereinstimmend eine Abfolge von miteinander im Wettbewerb stehenden Parteiformen identifizieren: Notablenpartei (das ist Webers Honoratiorenpartei), dann Weltanschauungspartei, dann Massenpartei oder, bei Kirchheimer: catch-all-party, schließlich Kartellpartei und heute Bewegungspartei. ${ }^{34}$ Wichtig für diesen Zusammenhang ist auch die Verbindung mit Duvergers Unterscheidung nach intern oder extern mobilisierten Parteien,

29 Siehe Lübbe-Wolff 2018.

30 Zuletzt hierzu Kielmansegg 2018; Lübbe-Wolff 2018.

31 Weber 1988 [1919], S. 500.

32 Ebd., S. 499.

33 Zitiert nach Lübbe-Wolff 2018, S. 139.

34 Katz, Mair 1995; Katz, Mair 1996; Katz, Mair 2009. 
Parteien also, die Zugang zur Ämterpatronage haben (intern mobilisiert) oder diesen Zugang nicht haben (extern mobilisiert). ${ }^{35}$

Weber selbst, das muss man gerechterweise betonen, ist sich völlig bewusst, in einer Übergangsphase zu leben. Er konzediert, dass die »künftige Gestaltung der Parteien [...] noch ganz im Dunkel [liegt]. Es ist daher heute noch in keiner Weise zu übersehen, wie sich äußerlich der Betrieb der Politik als >Beruf gestalten wird « ${ }^{36}$ Insofern ist es ihm dann auch nachzusehen, wenn sich seine zentrale Diagnose von den zwei grundsätzlichen Entwicklungsmöglichkeiten nicht bestätigt hat: »Aber es gibt nur die Wahl: Führerdemokratie mit >Maschineく oder führerlose Demokratie, das heißt: die Herrschaft der Berufspolitiker ohne Beruf, ohne die inneren, charismatischen Qualitäten, die eben zum Führer machen. (Und das bedeutet dann das, was die jeweilige Parteifronde gewöhnlich als Herrschaft des >Klüngels< bezeichnet). «37

An dieser Stelle ist zu betonen, um wie viel leichter es heute fällt zu sehen, was Weber nicht sah - und dass sich dies nicht als Ausweis eines heute irgendwie größeren politischen Weitblicks oder einer auch nur irgendwie ausgeprägteren demokratischen Reife verstehen lässt. Was dies allenfalls vor Augen führt, ist das dramatische Ausmaß an Nichtwissen, unter dem die zentralen politischen und auch wissenschaftlichen Akteure damals handelten und wohl grundsätzlich immer handeln müssen (denn es gilt ja gleichermaßen für unsere Gegenwart, dass wir nicht sehen können, was wir nicht sehen können). Und es wäre zumindest eine Warnung davor, die Demokratie, die Weber vermutlich mit der Brille von Droysen und Mommsen betrachtete, heute mit der Brille Webers zu betrachten.

Was wissen wir heute, was Weber nicht wusste und teilweise auch nicht wissen konnte? In aller Kürze: Das 1918 eingeführte Verhältniswahlrecht machte die Parteien überhaupt zum ersten Mal zu verpflichtungsfähigen und strategisch handlungsfähigen Akteuren. ${ }^{38} \mathrm{Im}$ exakten Gegensatz zu Webers Diagnose vom »Banausenparlament « als seiner Folge war es die Voraussetzung für einen Wettbewerb um politische Führungsämter - denn nun konnten die jeweiligen Parteieliten (der bürgerlichen Parteien, für die Sozialdemokratie als Paria-Partei des Kaiserreichs und deswegen als extern mobilisierte Partei stellt sich der Zusammenhang anders dar) erstmals überhaupt über geschlossen auftretende Fraktionen »herrschen « und damit parlamentarisch für ideologisch-programmatisch klar umrissene Positionen eintreten und auch elektoral für sie werben.

Dafür erwies sich nicht, contra Weber, Ämterpatronage als Anreizinstrument von zentraler Bedeutung, sondern die Androhung einer ausbleibenden Wiedernominierung als wichtigstes Sanktionsmittel der Parteieliten gegenüber den backbenchers/Hinterbänklern als den gewöhnlichen Mitgliedern des Reichstags. Dieses Mittel bekamen die Parteiführer erst mit der Verhältniswahl und der damit einher-

35 Shefter 1994 a; Shefter 1994 b.

36 Weber 1992 [1918], S. 60.

37 Ebd., S. 59.

38 Schröder, Manow 2014; Schröder, Manow 2020. 
gehenden zentralen Kontrolle über die Listenerstellung in die Hand. Das war dann auch die Voraussetzung der Ausbildung von Regierungs-Oppositions-Dynamiken, wie sie sich durch das Mehrheitswahlrecht in Kombination mit der Agendamacht der parlamentarischen Mehrheit in Großbritannien seit Ende des 19. Jahrhunderts etabliert und dort zur schrittweisen Parlamentarisierung der britischen konstitutionellen Monarchie geführt hatten. ${ }^{39}$ Es waren diese Dynamiken zwischen Regierung und Opposition, die im House of Commons einen Prozess der politischen Führungsauslese antrieben, den Weber im britischen Fall so bewunderte - und der genau durch jene Weimarer Konstellation, für die Weber so machtvoll gekämpft hatte, mit dem direkt gewählten Reichspräsidenten und seinen gegenüber dem Parlament umfassenden Auflösungs- und Notstandsvollmachten verhängnisvoll konterkariert wurde. ${ }^{40}$

In dieser Perspektive zeigt sich schon Webers Diagnose von der im Kaiserreich ausbleibenden Parlamentarisierung als revidierungsbedürftig ${ }^{41}$ - mit der Nebenbemerkung, dass seine Interpretation bis heute gängige Münze der Geschichtswissenschaft geblieben ist. Es ist wenig überzeugend, die ausbleibende Parlamentarisierung erneut entweder als reines Mentalitäts- oder Charakterproblem darzustellen - das Führungspersonal in den Parteien und im Parlament sei halt dürftig gewesen, mutlos, es hätte politische Feigheit vorgeherrscht, das deutsche Bürgertum hätte sich beflissen dem Bismarck'schen Cäsarismus unterworfen, auch aus Angst vor einer mit der Parlamentarisierung zwangsläufig einhergehenden Zunahme des Einflusses der Sozialdemokratie - oder aber, alternativ, auf die angebliche Machtlosigkeit des Reichstags abzustellen und insofern eine schlichte (und dann auch noch falsche) Tautologie zu formulieren: Dass der Reichstag in dem Gefüge von Kaiser, Preußen, Bundesrat und Reichskanzler so machtlos gewesen sei, habe verhindert, dass politische Akteure gegenüber der Reichsführung glaubwürdig einen eigenständigen Führungsanspruch des Parlaments hätten formulieren können, weswegen der Reichstag im politischen Gefüge des Kaiserreichs so machtlos geblieben sei.

Solange unter dem absoluten Mehrheitswahlrecht die politischen Parteien im Wilhelminischen Reichstag lockere Honoratiorenbünde blieben, die - gerade aufgrund der Heterogenität lokaler Wahlkreisabsprachen ${ }^{42}$ - nicht zu einem geschlossenen Handeln fähig waren, machte es für die ja nur sehr eingeschränkt handlungsfähigen Parteieliten überhaupt keinen Sinn, vis-à-vis der Regierung

39 Cox 1987; Dewan, Spirling 2011; Eggers, Spirling 2018.

40 Als weiterer Aspekt, der bei Weber nicht vorkommen konnte, wären in diesem Zusammenhang die sehr geringen Wahlhürden des Weimarer Verhältniswahlrechts zu nennen, die zur Parteienfragmentierung führten und keine Anreize zur parlamentarischen Kompromissbildung setzten (Schröder, Manow 2016). Erneut ist zu betonen, dass dies nicht mit der Diagnose »mangelnde Kompromissbereitschaft " auf einen schlichten Charakterdefekt der beteiligten Akteure zurückzuführen ist - wie es voluntaristische Interpretationen bis heute nahelegen -, sondern dass wir es mit systematischen institutionellen Anreizwirkungen zu tun haben; es macht sich - damals, wie heute - »ein fataler Mangel an Institutionenverstand « (Lübbe-Wolff) bemerkbar.

41 Weber 1988 a [1917].

42 Reibel 2007; Reibel 2011. 
einen parlamentarischen Machtanspruch zu formulieren, den man unter den gegebenen Umständen dann doch nicht einlösen konnte. Aus Sicht der Parteieliten war es viel rationaler, auf die bereits föderal abgestimmten Initiativen der Reichsregierung zu warten und dann je nach Ad-hoc-Mehrheitslagen auf partielle Änderungen der Gesetzesentwürfe zu drängen, anstatt selbst initiativ zu werden. Das ist unter »Feigheit « nicht zu rubrizieren.

Diese Zusammenhänge verdeutlichen, so denke ich, die Bedeutung der Wahl und des Wahlrechts für die innere Verfasstheit der Parteien, die Weber systematisch entgehen. Er betont zwar völlig zu Recht, dass sie als voluntaristische, im freien Wettbewerb miteinander stehende, im Wesentlichen extra-konstitutionelle Institutionen zu verstehen seien und dass damit der staatlichen Beeinflussung ihrer Verfasstheit deutliche Grenzen gesetzt sind. ${ }^{43}$ Dass sie sich aber selbst - vermittels des Wahlrechts - eine spezifische Organisationsform geben und dass dies von alles überragender Bedeutung für die Funktionsweise der repräsentativen Demokratie ist, entgeht Weber. Bei aller scharfen, ätzenden Polemik gegenüber den Honoratiorenparteien vermag Weber nicht zu sehen, dass genau dieser Parteientypus das Resultat bestimmter wahlrechtlicher Anreizmechanismen war, nämlich des absoluten Mehrheitswahlrechts des Kaiserreichs mit seinem Vorherrschen zunehmend heterogener Wahlkreisabsprachen, ${ }^{44}$ die das Verhältniswahlrecht, 1918 beschlossen, 1919 erstmals angewendet, nun gerade beseitigte.

\section{Ausblick}

Was sind die Lehren, die wir heute daraus ziehen können? Wir wissen, dass sich die Organisationsform politischen Kollektivhandelns namens »Partei « heute in einer Krise befindet. Die Gründe sind vielfältig - sie auch nur zu skizzieren würde den Rahmen sprengen. ${ }^{45}$ Damit wird auch zunehmend sichtbar, dass ein politisches System der »repräsentativen Demokratie «, dessen Funktionieren kritisch auf der modernen politischen Partei basiert, zusammen mit der Organisationsform Partei in die Krise gerät. ${ }^{46}$ Der politische Populismus ist ein Indikator dieser Krise. Hier nun erneut auf »Haltungen « und »Einstellungen « zu rekurrieren oder auf Werteappelle als "Abwehrzauber gegen links und rechts ${ }^{47} \mathrm{zu}$ setzen, also zu denken, das Problem bestünde wesentlich in einem Mangel an gutem Willen echter Demokraten - das ließe sich mit Weber wohl zu Recht als »Tintenfassromantik « charakterisieren. Aber selbst wenn man mit ihm insoweit übereinstimmen würde, dass eine kritische Analyse der Demokratie damals wie heute eine Institutionenanalyse sein muss, lassen sich durch den Blick zurück auf Webers Position in den

43 Weber 1988 b [1917].

44 Schröder, Manow 2014; Schröder, Manow 2020.

45 Synthesen der einschlägigen Befunde finden sich unter anderem bei Mair 2013; Torney 2015.

46 Manow 2020; Meinel 2019.

47 Meinel 2019, S. 7. 
politischen Umbruchjahren 1918/19 für unsere Lage keine »Eindeutigkeiten « gewinnen, sondern nicht mehr (aber auch nicht weniger) als ein geschärfter Sinn für die ungeheure »Komplexität der rechtlichen und außerrechtlichen Zusammenhänge « ${ }^{48}$ die in Fragen der Demokratie und ihrer Verfassung jeweils zu berücksichtigen wären.

\section{Literatur}

Bureau of the Census 1949. Historical Statistics of the United States 1789-1945. A Supplement to the Statistical Abstract of the United States. Washington: United States Department of Commerce.

Cox, Gary W. 1987. The Efficient Secret - The Cabinet and the Development of Political Parties in Victorian England. New York: Cambridge University Press.

Dewan, Torun; Spirling, Arthur 2011. "Strategic Opposition and Government Cohesion in Westminster Democracies", in American Policial Science Review 105, 2, S. 337-358.

Eggers, Andrew; Spirling, Arthur 2018. »The Shadow Cabinet in Westminster Systems: Modeling Opposition Agenda Setting in the House of Commons, 1832-1915 «, in British Journal of Political Science 48, 2, S. 343-367.

Gusy, Christoph 1993. Die Lehre vom Parteienstaat in der Weimarer Republik. Baden-Baden: Nomos.

Katz, Richard S.; Mair, Peter 1995. »Changing Models of Party Organizations and Party Democracy. The Emergence of the Cartel Party ", in Party Politics 1, 1, S. 5-28.

Katz, Richard S.; Mair, Peter 1996. "Cadre, Catch-all or Cartel? A Rejoinder ", in Party Politics 2, 4, S. 525-534.

Katz, Richard S.; Mair, Peter 2009. »The Cartel Party Thesis: A Restatement «, in Perspectives on Politics 7, 4, S. 753-766.

Kaube, Jürgen 2014. Max Weber. Ein Leben zwischen den Epochen. Berlin: Rowohlt.

Kielmansegg, Peter Graf 2018. „Der Reichspräsident - ein republikanischer Monarch? «, in Das Wagnis der Demokratie. Eine Anatomie der Weimarer Reichsverfassung, hrsg. v. Dreier, Horst; Waldhoff, Christian, S. 219-240. München: C. H. Beck.

Lethen, Helmut 1994. Verhaltenslehren der Kälte. Lebensversuche zwischen den Kriegen. Frankfurt a. M.: Suhrkamp.

Lübbe-Wolff, Gertrude 2018. "Das Demokratiekonzept der Weimarer Reichsverfassung ", in Das Wagnis der Demokratie. Eine Anatomie der Weimarer Reichsverfassung, hrsg. v. Dreier, Horst; Waldhoff, Christian, S. 111-149. München: C. H. Beck.

Mair, Peter 2013. Ruling The Void: The Hollowing of Western Democracy. London: Verso.

Manow, Philip 2020. (Ent-)Demokratisierung der Demokratie. Ein Essay. Berlin: Suhrkamp.

Meinel, Florian 2019. Vertrauensfrage. Zur heutigen Krise des Parlamentarismus. München: C. H. Beck.

Michels, Robert 1957 [1911]. Zur Soziologie des Parteiwesens in der moderen Demokratie: Untersuchungen über die oligarchischen Tendenzen des Gruppenlebens. 2. Auflage. Stuttgart: Kröner.

Momigliano, Arnaldo 1991 [1955]. Wege in die Alte Welt. Berlin: Wagenbach.

Mommsen, Wolfgang J. 1974. "Zum Begriff der >plebiszitären Führerdemokratie«", in Max Weber: Gesellschaft, Politik und Geschichte, hrsg. v. Mommsen, Wolfgang J., S. 42-71. Frankfurt a. M.: Suhrkamp.

Mommsen, Wolfgang J. 1974 [1959]. Max Weber und die deutsche Politik, 1890-1920. Tübingen: Mohr Siebeck.

Mommsen, Wolfgang J. 1989. "Politik und Politische Theorie bei Max Weber ", in Max Weber heute. Erträge und Probleme der Forschung, hrsg. v. Weiß, Johannes, S. 514-542. Frankfurt a. M.: Suhrkamp. 
Mommsen, Wolfgang J.; Schluchter, Wolfgang; Morgenbrod, Birgitt. Hrsg. 1992. Max Weber: Wissenschaft als Beruf (1917/1919), Politik als Beruf (1919). Max Weber Gesamtausgabe, Abteilung I: Schriften und Reden, Band 17. Tübingen: J.C.B. Mohr (Paul Siebeck).

Müller, Wolfgang C.; Strom, Kaare. Hrsg. 1999. Policy, Office or Votes? How Political Parties in Western Europa Make Hard Choices. Cambridge Studies in Comparative Politics. New York: Cambridge University Press.

Nippel, Wilfried 2008. Antike oder moderne Freiheit? Die Begründung der Demokratie in Athen und in der Neuzeit. Frankfurt a. M.: Fischer.

Reibel, Carl-Wilhelm (Bearbeiter) 2007. Handbuch der Reichstagswablen 1890-1918: Bündnisse, Ergebnisse, Kandidaten. Düsseldorf: Droste.

Reibel, Carl-Wilhelm 2011. »Bündnis und Kompromiss: Parteienkooperation im Deutschen Kaiserreich 1890-1918 ", in Historische Zeitschrift 293, 1, S. 71-114.

Schivelbusch, Wolfgang 2001. Die Kultur der Niederlage: Der amerikanische Süden 1865. Frankreich 1871. Deutschland 1918. Hamburg: Rowohlt.

Schmitt, Carl 2015 [1921]. Die Diktatur. Von den Anfängen des modernen Souveränitätsgedankens bis zum proletarischen Klassenkampf. Berlin: Duncker \& Humblot.

Schröder, Valentin; Manow, Philip 2014. »Elektorale Koordination, legislative Kohäsion und der Aufstieg der modernen Massenpartei: Die Grenzen des Mehrheitswahlrechts im Deutschen Kaiserreich, 1890-19181 «, in Politische Vierteljahresschrift 55, 3, S. 518-554.

Schröder, Valentin; Manow, Philip 2016. »Too Clever by Half? Low-threshold Closed-list Proportional Representation and Party Split in Weimar Germany, 1919-1930«. Workshop »The Politics of Parliamentarization «, University of Bremen, 14-15 July 2016. CES Open Forum Series.

Schröder, Valentin; Manow, Philip 2020. "An Intra-Party Account of Electoral System Choice", in Political Science Research and Methods 8, 2, S. 251-267.

Schumpeter, Joseph A. 1993 [1942]. Kapitalismus, Sozialismus und Demokratie. Tübingen, Basel: Francke.

Shefter, Martin 1994 a. »Party and Patronage: Germany, England, and Italy «, in The State: Critical Concepts, III, hrsg. v. Hall, John A., S. 103-143. London, New York: Routledge.

Shefter, Martin 1994 b. "Patronage and Its Opponents: A Theory and Some European Cases", in Political Parties and the State. The American Historical Experience, hrsg. v. Shefter, Martin, S. 21-60. Princeton: Princeton University Press.

Torney, Simon 2015. The End of Representative Politics. London: Polity Press.

Weber, Max 1988 a [1917]. »Parlament und Regierung im neugeordneten Deutschland «, in Max Weber: Gesammelte Politische Schriften, S. 306-443. Tübingen: Mohr Siebeck.

Weber, Max 1988 b [1917]. "Wahlrecht und Demokratie in Deutschland ", in Max Weber: Gesammelte Politische Schriften, S. 245-291. Tübingen: Mohr Siebeck.

Weber, Max 1988 [1919]. »Der Reichspräsident «, in Max Weber: Gesammelte Politische Schriften, S. 498-501. Tübingen: Mohr Siebeck.

Weber, Max 1992 [1918]. Politik als Beruf. Stuttgard: Reclam. 
Zusammenfassung: In dem Beitrag wird insbesondere anhand des Vortrags Politik als Beruf Max Webers Verständnis von Demokratie in den Umbruchjahren 1918/19 diskutiert. Weber nähert sich seinem Thema nicht in der Überlieferungslinie einer Politischen Philosophie, die politische Repräsentation und die vertragstheoretische Begründung von Herrschaft in ihren Mittelpunkt stellt, sondern aus der Perspektive seiner Theorie der Modernisierung und des okzidentalen Rationalismus, die unter anderem die Berechenbarkeit des modernen Anstaltsstaats mit seiner unabhängigen Fachbürokratie betont. Vor diesem Hintergrund wird demokratische Politik auf potenziell irrationales charismatisches Führertum und den Rückschritt in der Rationalisierung der Staatsverwaltung durch die Patronage und Beutepolitik der Parteien reduziert. Eine solche Sicht erwies sich in den Debatten über die Weimarer Reichsverfassung als verhängnisvoll.

Stichworte: Max Weber, Demokratie, Weimarer Reichsverfassung, Politik als Beruf, Parteienpatronage

\title{
Max Weber's Concept of Democracy
}

Summary: The article discusses Weber's understanding of democracy in Germany's years of turmoil 1918/19; in particular, he elaborated it in his lecture Politics as a Vocation. Weber does not approach his theme in the traditional way: as a question of Political Philosophy, of democratic representation or in the context of a contract-theory of political rule. Rather, he interprets democracy against the background of his theory of modernization and rationalization, emphasizing the contribution of the modern state bureaucracy to the regularity, predictability, and legality of power. In this perspective, democratic politics is reduced to potentially irrational charismatic leadership and the regression in the rationalization of state administration by party machines with their patronage and prey politics. Such a perspective proved disastrous in the debates over Weimar's constitution, in which Weber played an important rule.

Keywords: Max Weber, democracy, constitution of the Weimar Republic, Politics as a Vocation, party patronage

\author{
Autor \\ Philip Manow \\ SOCIUM \\ Universität Bremen \\ Mary-Somerville Str. 5 \\ 28359 Bremen \\ Deutschland \\ manow@uni-bremen.de
}

